\title{
Sea gliders piloted by a centralised mission management system
}

\author{
Magali BARBIER, \\ Eric BENSANA, \\ David DOOSE \\ ONERA/DTIS \\ Toulouse, FRANCE \\ Firstname.Surname@onera.fr
}

\author{
Florent BESSON, \\ Jonathan ROMERO \\ ALSEAMAR \\ Rousset, FRANCE \\ InitialfirstnameSurname \\ (a)alseamar-alcen.com
}

\author{
Mikaël LEOPOLDOFF \\ Stéphan LARRASOAIN \\ CENTRAL WEB \\ La Valette Du Var, FRANCE \\ InitialfirstnameSurname \\ (a)centralweb.fr
}

\begin{abstract}
The objective of AGLIMMS project, whose acronym stands for Acoustic GLIders Mission Management System, was to coordinate a fleet of underwater gliders whose missions are to obtain physical, chemical, biological and/or acoustic measurements on a large 3D sea area. This paper introduces components and tests of the developed planning and supervision system that was integrated in a global centralised architecture and describes the result of sea experimentation conducted in November 2019 in the Mediterranean Sea with three SeaExplorer gliders from Alseamar.
\end{abstract}

Keywords-underwater glider, fleet, mission management system, navigation, planning, supervision, sea experimentation

\section{I.INTRODUCTION}

Oceanographic missions performed by underwater gliders are various [1]: climate change monitoring, analysis of human activity (oil and mining industries), study of local flora and fauna, seabed mapping for its exploitation and protection...

Whereas missions assigned to most Autonomous Underwater Vehicles last several days on several square dozen kilometres, underwater gliders can stay at sea for several months and scan thousands of kilometres. These lowcost vehicles move silently while interfering very little with their environment and their low weight allows light deployments and recoveries. Their drawbacks are a bad underwater positional accuracy in hundreds of meters and their limited power capacity, a few watts used for travelling (by changing buoyancy and using winged surfaces to produce a sawtooth-like trajectory) and for payload. When in mission, they surface every few hours for measurement data transmission and potential trajectory update. They are controlled by an experienced pilot who is alerted each time a glider surfaces and who can adapt the trajectory or change the mission if needed.

Using a fleet (sometimes called a network) of gliders either increases the spatial acquisition or reduces the processing time. The probability to detect sources of signals (gas leak, marine mammals, anthropogenic noise...) increases and positional accuracy is better thanks to a possible triangulation. Specific formations such as comb allow for a better coverage of spatial areas. Lastly, fleet missions can potentially proceed when a glider leaves the team, especially when reconfiguration issues are managed.
For an efficient coordination, the management of a fleet of gliders however requires more than individual experienced pilots. Many types of heterogeneous data must be assimilated: they stem from platforms, from the run of models (e.g. currents, sound propagation) and from external world (e.g. automatic ship identification systems AIS, bathymetry, weather). A centralised global Mission Management System (MMS) is thus required to help the pilots, by leaving them only high-level tasks while algorithms perform the coordination activities.

Four types of underwater gliders are on the market: SeaExplorer from Alseamar, Seaglider from University of Washington, Spray from Scripps Institution of Oceanography, and Slocum from Teledyne Webb Research. Several fleet experiments [2] [3] [4] [5] have been achieved with a fleet of above gliders, which were either individually operated by pilots discussing together or virtually moored.

Section II presents AGLIMMS project in which this work falls. Section III develops the components of the Planning and Supervision System (PSS). Section IV shows evaluation tools and simulation results. Section $\mathrm{V}$ reports the experimentation results then section VI feedback and perspectives.

\section{AGLIMMS PROJECT}

Three complementary partners were involved in the French AGLIMMS project (2016-2019): Alseamar (project lead) operate SeaExplorer (Fig. 1) since 2017; ONERA do extensive research on unmanned vehicles cooperation since 2007; CentralWeb develop big data management and webmapping systems.

The objective of AGLIMMS was to build a centralised MMS to coordinate efficiently a fleet of gliders. This paper sums up the developed Planning and Supervision System [6] and focuses on sea experimentation conducted during twelve days in Mediterranean Sea in November 2019 with three SeaExplorer. MMS is centralised on a web-based platform called GLIMPSE (GLIder Mission Piloting System) that allows multiple users to prepare, supervise and analyse gliders missions [7]. 

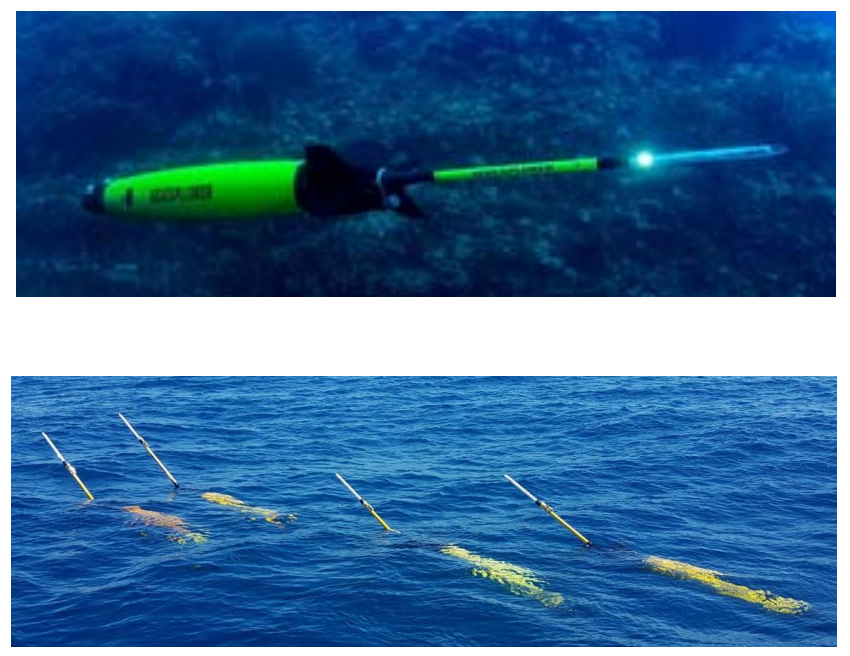

Fig. 1. SeaExplorer underwater gliders from Alseamar.

\section{PlanNING AND SUPERVISION SYSTEM (PSS)}

AGLIMMS PSS aimed at managing the coordination of all gliders actions for the achievement of missions in an optimised way.

Fleet mission defined by an operator contains the navigation area, a set of gliders, several phases of operation, and constraints between phases. Five types of phases are available amongst them path following (a path being defined by a sequence of waypoints), systematic coverage of an area for data gathering, and random coverage of an area for signal detection. Environment data consist of 3D sea currents maps, bathymetry maps and potential forbidden areas.

Fig. 2 shows interactions between PSS components within the MMS. Inputs are the mission (initial and possibly upgraded during its execution) and data frames sent by gliders when on surface. Outputs are controls sent to gliders (a sawtooth-like navigation profile towards next surfacing point) and fleet plans sent to the GLIMPSE user interface.

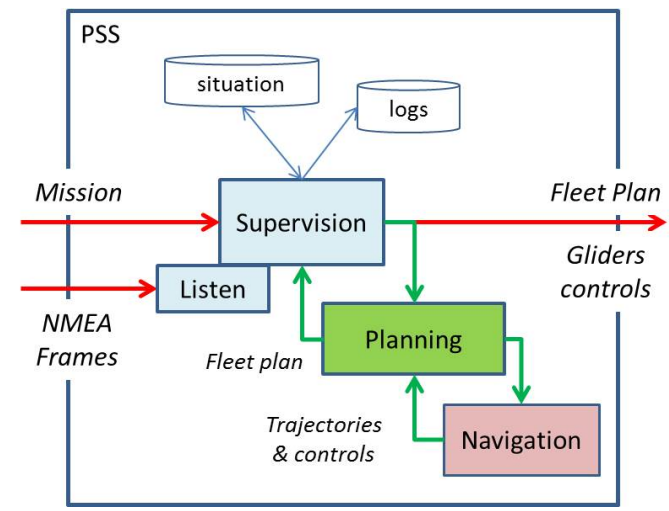

Fig. 2. Planning and Supervision System.

Three functions interact in PSS:

- Supervision manages transitions between phases (assuming one phase is finished when all gliders ended it), triggers planned gliders control actions at computed diving times and follows their execution. When a glider surfaces, it checks the current situation and selects next appropriate steps: triggering a glider dive, updating glider navigation plan, updating fleet mission plan. It can then call the planning to enhance a plan; as a general rule, any evolution of situation can trigger the planning;

- Planning computes a fleet plan composed of all individual glider plans. A glider plan or itinerary is one sequence of all glider control actions from now up to end of mission or to a specific date. It considers mission constraints and calls the navigation function to evaluate individual trajectories cost; criteria relate to duration, time interval, consumption, and distance;

- Navigation computes all surfacing actions between two surfacing waypoints while considering gliders characteristics and environmental constraints; interlacing between planning and navigation functions is detailed in [6].

Three threads compose supervision: monitoring of asynchronous events reception, connection to Alseamar communication server and reader of gliders frames. All data are in JSON format. External and internal events activate situation update and recording. Strategies allow the treatment of requests arrival among them operator requests: new mission, mission update, plan, and stop mission.

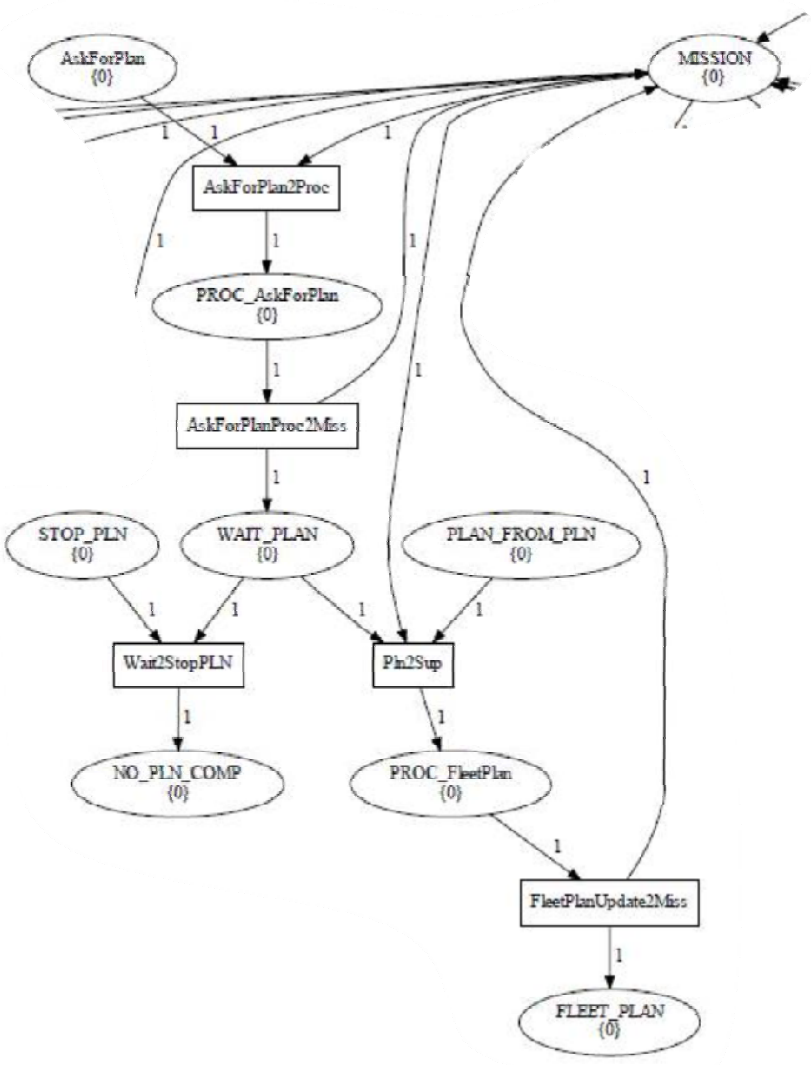

Fig. 3. Petri net part modelling replanning process in supervision.

Interpreted Petri nets based on SNAKES Python library [9], a part of which being automatically generated, manage state changes in supervision code. A Petri net [8] is a bipartite graph with two types of nodes: places model the state of the system and transitions model its evolution. As one example, Petri net on Fig. 3 shows replanning process: 
- When mission is ongoing (one token in MISSION place) and a plan requested whatever its origin (one token in AskForPlan), transition AskForPlan2Proc is triggered, PROC_AskForPlan is processed mainly calling planning process then supervision waits for planning results (one token in WAIT_PLAN place);

- If a fleet plan is received (one token into PLAN FROM PLN), transition Pln2Sup is triggered and $\mathrm{PROC}$ FleetPlan is processed mainly storing the plan; place FLEET PLAN allows to count the number of computed plans while mission continues with updated actions;

- If no fleet plan is available given supervision criteria (one token into STOP PLN), supervision counts the failure (in NO_PLN_COMP) while reacting in consequence in program code given several possible strategies.

Planning problem, modelled as a Constraint Satisfaction Problem (CSP), is solved using the CHOCO tool [10]. Inputs are current state of the mission relative to gliders and phases. The algorithm selects phases to perform, assigns gliders to phases, chooses the sequence of phases visited by each glider, formation and splitting for each phase and computes surfacing waypoints to answer mission constraints. Problem variables are start end dates of each phase. Four ending cases appear when optimisation stops in the allocated time:

- In the ideal case, it finds a solution with proved optimality;

- In the common case, it finds a solution without proved optimality;

- It proves that no solution exists, needing some input constraints to be relaxed;

- It does not found any solution mainly due to an inconsistency in the given mission / situation.

Trajectories computed by navigation are gliders controls of the sawtooth-like navigation profile composed of vertical speed $\mathrm{Vz}$, slope $\gamma$, bottom immersion $\mathrm{Zb}$, top immersion $\mathrm{Zt}$ (as a glider does not surface after each ascent) and number of descent-ascent patterns $\mathrm{Sr}$ (Fig. 4). Mission area is meshed in cells with $2 \mathrm{D}$ bathymetry, 3D currents (direction and speed by depth layers) and forbidden data. Several implemented algorithms give a quick path, an optimal path, successive better paths, these solutions either locally or globally overall the area.

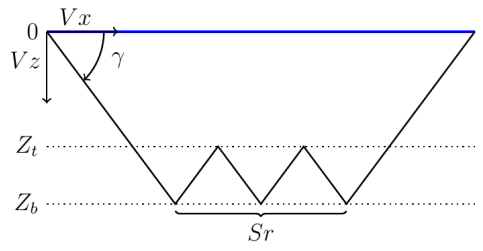

Fig. 4. Sawtooth-like navigation profile of a glider.

\section{PSS SIMULATION TESTS}

Several tools enabled testing PSS, first by simulation then at sea:
- A Common Gateway Interface (CGI) allows an operator to send requests to PSS thanks to a web page (Fig. 5);

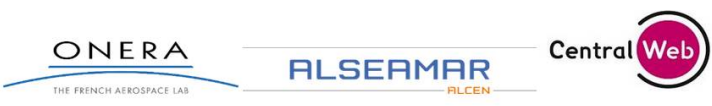

CGI web page for AGLIMMS Planning and Supervision System

PSS requirements: $>$ Windows $7,>$ jdk-12.0.1, $>$ python 3.7 .4 , libraries to install: SNAKES requests psutil geopy Load a new mission: Parcourir... Aucun fichier sélectionné. Send Mission File

Ask for a fleet plan: Ask for a fleet plan

Update a mission: Parcourir... Aucun fichier sélectionné. Send Updated Mission File

Stop mission for all gliders: Send Stop

Emulate NMEA frames for: SEAONERA4 SEAONERA5 SEAONERAG Init All Gliders

Fig. 5. Simplified version of CGI interface.

- An itinerary viewer shows fleet plans computed by planning (Fig. 6): green points are gliders surface positions (underwater would be in blue), mission is to rally the centre of the East square area, to perform there a random coverage, to join the North-West point of the coverage area and to return to a West recovery point with a comb formation; figure gives itineraries (green) and trajectories (black);

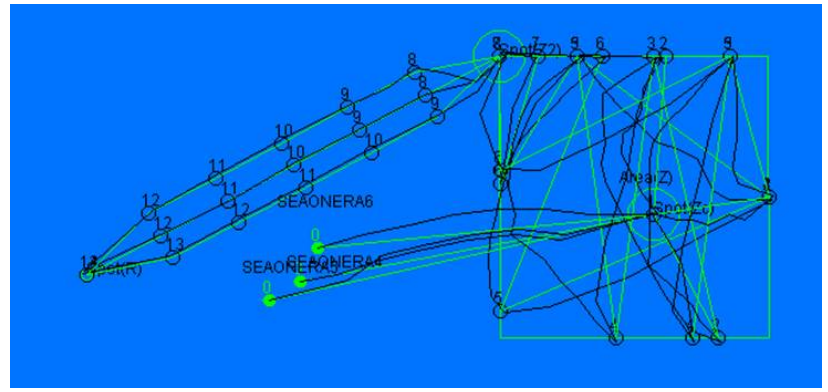

Fig. 6. Fleet plan viewer.

- A viewer shows 2D and 3D trajectories (Fig. 7): top image highlights the avoidance of low isobaths, bottom image gives associated navigation profile;
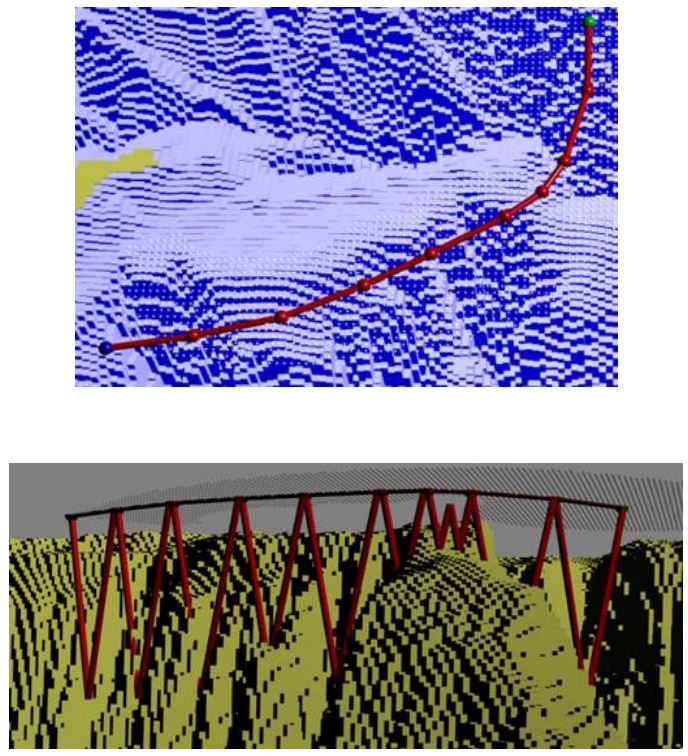

Fig. 7. 2D and 3D trajectory viewer. 
- A glider Alseamar tool simulates its surfacing with spatial drift according to received controls.

Several simulated missions allowed to evaluate PSS components and to prepare sea experimentation: 1 to 3 gliders, 2 to 10 days, no formation, comb and triangular formations. Navigation tests on several areas, with distances between 10 to $100 \mathrm{~km}$, and various currents orientations and strengths, allowed tuning navigation parameters.

These simulations highlighted PSS capacity to consider missions with 2 to 4 sequential phases, three homogeneous gliders, energy minimisation, a few operator events and direct behavioural reactions. For scale, Fig. 8 zooms in the area equivalent to 35 days of simulation for the fleet (one colour per glider).

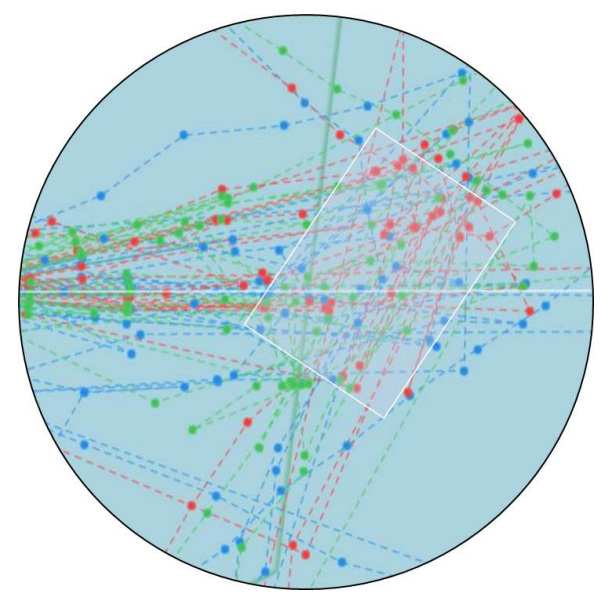

Fig. 8. GLIMPSE view of 35 days of simulation tests.

Several figures also show added PSS results:

- Planning of fleet plan with different phases in Fig. 6;

- Planned waiting strategy to perform a comb formation in Fig. 9 (horizontal black lines stand for round navigation profiles);

- Simulated achievement of a comb formation displayed on GLIMPSE in Fig. 10 (with spatial drift).

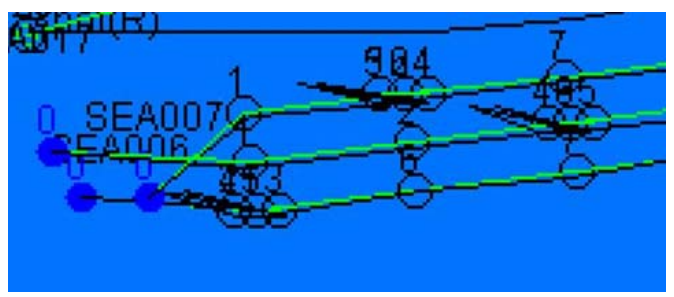

Fig. 9. Waiting strategy for comb formation.

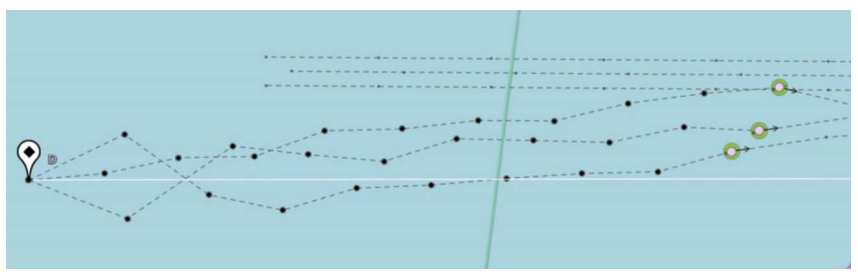

Fig. 10. Simulated comb formation.
Due to focus on sea experimentation and lack of fast time simulator, there was however not enough time to evaluate different navigation criteria, planning optimality and a larger set of disruptive events in supervision.

\section{EXPERIMENTATION RESULTS}

Simulation and sea tests with whole PSS, reported below, highlighted the good behaviour of each individual component and of the system integration within MMS: plans computed in most cases were consistent and events correctly supervised.

\section{A. First sea tests with one glider}

October 2019, first tests with a SeaExplorer allowed the validation in real conditions of main supervision capabilities: frame receipt, frame filtering, situation update, planning and navigation calls, plan receipt, situation update, and control sending. Fleet plan displayed on GLIMPSE were also correct.

\section{B. Sea mission}

Mission area (Fig. 11) for the three SeaExplorer is close to Mediterranean coast (facilitating deployment and recovery), has various current strengths (especially the coastal Ligure current) and is quite outside traffic AIS.

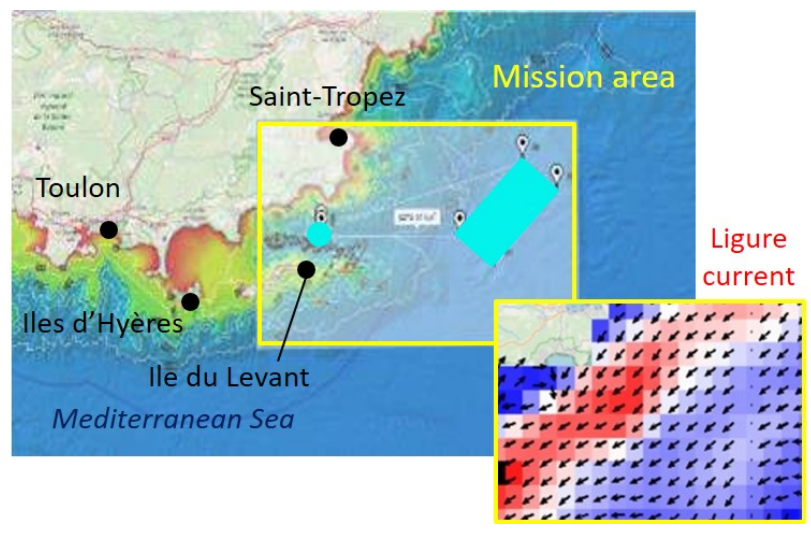

Fig. 11. Sea mission: bathymetry and current (first day).

Initial scenario for the team of gliders was composed of four phases: rally a desired coverage area $(\sim 25 \mathrm{~km}$, comb formation with a $1 \mathrm{~km}$ space between gliders, immersion 0 $700 \mathrm{~m})$, perform a random coverage $\left(180 \mathrm{~km}^{2}, 36 \mathrm{~h}\right.$, immersion $0-350 \mathrm{~m})$, rally the North point then the recovery point $(25 \mathrm{~km}$, immersion $0-700 \mathrm{~m})$. On Fig. 11 , coverage area is the light blue rectangle whereas start and recovery positions are the light blue circle.

\section{Step by step experimentation}

Day 0: 20 November 2019. Pilots performed some gliders balancing at sea to stick PSS navigation model. We ran PSS and first planning computation gave a duration of 9 days for this mission, letting about one third of energy for each glider at mission end. Due to local drifts, two gliders received specific controls to wait for the third one (as in Fig. 9). Pilot validated first computed controls together with the global obtained plan.

Days 1-3. Early difficulties were that gliders surfacing locations were quite opposite to given head courses. The bad 
weather explained this behaviour: a remarkable event occurred between 22 and 24 November, the equivalent of 3 months' rain fell in 48 hours in the lands at about $70 \mathrm{~km}$ from the gliders location. Surface currents in opposite direction of course reversed their moves. PSS could not adapt to this type of rare event, only suffering its consequences. Moreover, drifts increased due to controls' checking by pilot and internal glider's process before diving. Gliders finally drifted on Mago sandbank (immersion lower than 100m) and PSS found no navigation solution. Even modifying mission had no effect: the targeted coverage area was unreachable. We finally stopped PSS and pilot skilfully led gliders out of the sandbank then towards the coverage area with adequate controls (gliders most often in deep-water at a $700 \mathrm{~m}$ immersion). Fig. 12 shows gliders drift during these days (black lines).

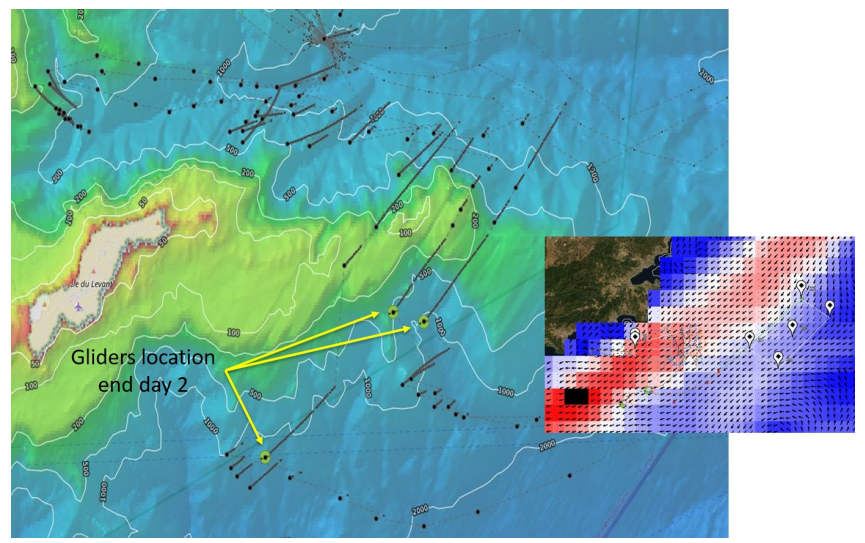

Fig. 12. Gliders surface drift (days 2-3).

Days 4-7. We updated mission to deal with the experimentation period, mainly with a shorter coverage area $\left(\sim 107 \mathrm{~km}^{2}\right)$. Its random coverage lasted about 3 days. Fig. 13 highlights differences between controls computed by PSS and real controls (left) and the global observed drift toward West (right), mainly due to insufficiently accurate current model used by PSS.

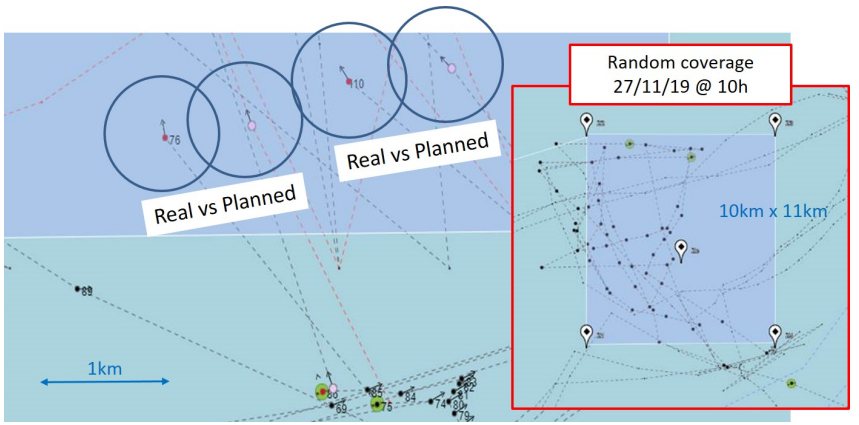

Fig. 13. Real vs planned surface locations for two gliders (left) and random coverage (right).

Days 7-9. Due to good PSS running during previous coverage and the little current at the gliders location, we added to mission a rallying phase with a comb formation. Fig. 14 shows this not-so-easy-to-follow rallying.

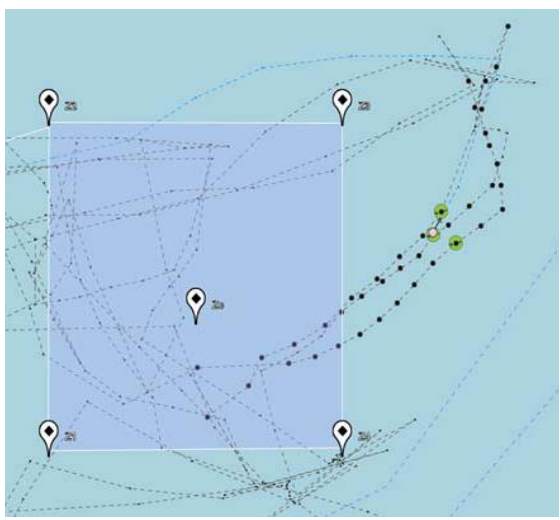

Fig. 14. Comb formation rallying.

Days 9-11. Rallying a recovery area going through an intermediary area added to mission allowed gliders to be carried by the current then speeded up this return phase.

Day 12. We stopped PSS and pilots organised the recovery of gliders.

Fig. 15 shows gliders surfacing points during November 2019 experimentation (one colour per glider) amongst them 6 days continuously piloted by PSS. Gliders flied 300 to $330 \mathrm{~km}$ achieving about 200 descent-ascent patterns. Fig. 16 shows the surface currents for one glider.

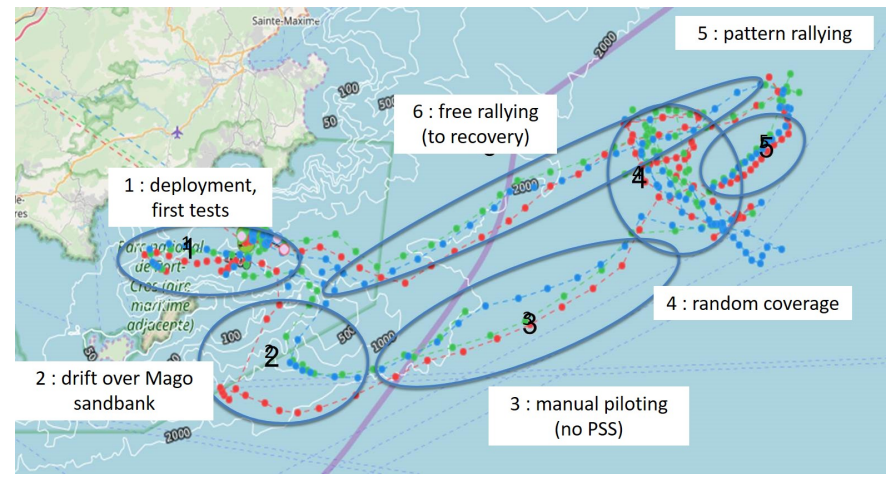

Fig. 15. Gliders surfacing points during the twelve experimentation days.

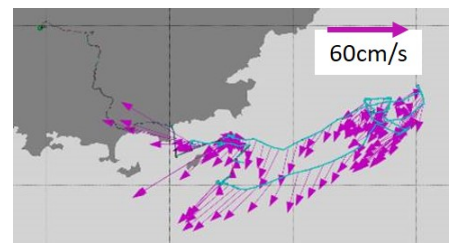

Fig. 16. Surface currents for one glider during experimentation.

\section{Experimentation feedback}

This large and intense experimentation was full of positive feedback. PSS did well, and we even enhanced PSS functions and added some new functionalities (like real time mission update) during these tests achieved with real gliders moving in a real environment.

PSS navigation computed many trajectories: those applied by gliders but also a lot during the planning optimisation process. Sea experimentation highlighted difficulties for gliders to follow these controls in real environment. To go further, pilots could give more degrees 
of freedom to PSS to let it better optimise the trajectory (especially adding vertical speed and slope); it was difficult to maintain a formation flight with gliders controlled in position. PSS could also online update the hydrodynamic model to avoid some local optimistic anomalies observed in initial model. Using better currents data and even updating them online is also a challenge, as better models would give better solutions.

PSS planning computed many fleet plans for gliders coordination. A few strategies were tested at sea; several reasons explained the cases when it found no plan: mission or phase too short, lack of energy, no trajectory... This function offers much potential for mission management, just to mention parallel phases, loop phases, variety of coverages, of criteria, heterogeneous gliders / payloads... PSS could also select which gliders to assign to phases, and order phases itself.

PSS supervision managed correctly all data exchanges, stored situation and triggered replanning process during six full days. Other strategies in reaction to new events would allow PSS to deal with high-level operator requests, environment data, critical glider alarm, signal detection... Adding generic Petri net patterns could enhance this function. A mission manipulation language would help managing plans with different decision levels. Again, PSS could interactively react with pilots for mission creation while checking mission consistency, proposing behavioural solutions...

Global feedback is that PSS decreased the pilot workload and allowed some fleet coordination. However, this experimentation also highlighted the huge potential of this type of centralized system and the work still to be done to have a validated qualified system proven in operational environment. Available spatial simulator helped preparing the experimentation, but it is not adapted to an evaluation process with many realistic test cases to answer main generic questions: which algorithms to increase spatial coverage, detection probability, and positional accuracy, which strategies to react better to this type of event... Especially with a mission duration expressed in months, an accelerated simulator is required. Tools should be available in the simulator to define scenarios, generate disturbing events, analyse runs, and replay them. The simulator should simulate constraints and uncertainties regarding to gliders dynamic, payloads, environment, and communication.

\section{CONCLUSIONS AND FUTURE WORK}

Project objective has been reached: in November 2019, the Planning and Supervision System PSS integrated in a global centralised architecture piloted a fleet of three SeaExplorer gliders in the Mediterranean Sea during about twelve days. GLIMPSE interface displayed fleet plans to pilots in real time and recorded all experimentation data.

Tests performed in a real environment showed some limits of the system, especially facing remarkable weather events; such data could be collected in real time and used by PSS to enhance reactive behaviours. Under usual weather conditions, PSS managed the mission in a consistent way and experimentation analysis explained observed deviations; proposed solutions thanks to this feedback require new studies.

Further work could enhance PSS capabilities to consider heterogeneous gliders and various payloads, generic team strategies and in relation communication strategies, and even onboard implementation of some decisional autonomy.

To complement mathematically proof of robustness studies, a full-featured simulator would allow algorithms and strategies evaluation in many test cases while keeping pilots in the loop at a high decision level in preparation and in execution stages.

This demonstration took place thanks to a strong collaboration between Alseamar, CentralWeb and ONERA's teams. Each partner showed off its skills and learnt from others. A new project might emerge to increase in particular PSS maturity.

\section{ACKNOWLEDGEMENT}

The French defence procurement agency DGA partially founded this work.

\section{REFERENCES}

[1] Rudnick, DL, 2016: Ocean research enabled by underwater gliders. Annu. Rev. Mar. Sci., 8, 519-541, doi:10.1146/annurev-marine122414-033913.

[2] Fratantoni, DM, 2005: Development of oceanographic sampling networks using autonomous gliding vehicles and demonstration of WHOI glider fleet operations in the tropical western Pacific for the Naval Oceanographic Office. Research gate.

[3] Leonard, NE, Paley, DA, Davis, RE, Fratantoni, DM, Lekien, F, Zhang, F, 2010: Coordinated control of an underwater glider fleet in an adaptive Ocean sampling field experiment .in Monterey Bay. Journal of Field Robotics 27(6), 718-740, doi: 10.1002/rob.20366.

[4] Real-Arce, DA, Quevedo, E, Barrera, C, Hernandez, J, Llinas, O, Barbero, F, Morais, A, 2016: A new integrated border security approach: the FP7 PERSEUS project. Marine Technology Society Journal, Volume 50, Number 4, July/August, pp. 14-25(12), doi: 10.4031/MTSJ.50.4.12.

[5] Onken, R, Fiekas, HV, Beguery, L, Borrione, I, Funk, A, Hemming, $M$, et al., 2018: High-resolution observations in the western Mediterranean Sea: the REP14-MED experiment. Ocean Sci., 14, 321-335, doi: 10.5194/os-14-321-2018.

[6] Barbier, M, Bensana, E, Doose, D, Besson, F, Le page, Y, Leopoldoff M, 2019: A mission management system for a fleet of gliders. OCEANS 2019, 18-20 juin 2019, Marseille, France, doi: 10.1109/OCEANSE.2019.8867580

[7] Besson, F, Pasqueron, O, Romero, J, Barbier, M, Bensana, E, Doose, D, Leopoldoff, M, and Larrasoain, S, et al, 2019: A centralized webbased platform for combined glider and satellite observation analysis. OCEANS 2019, doi: 10.1109/OCEANSE.2019.8867287.

[8] Murata, T. (1989). Petri nets: properties, analysis and applications. IEEE. 77(4), 541-580, doi:10.1109/5.24143.

[9] Pommereau, F., 2015. SNAKES: a flexible high-level Petri nets library. Proceedings of PETRI NETS'15. LNCS 9115, Springer 2015. doi: $10.1007 / 978-3-319-19488-2 \quad 13$.

[10] Prud'Homme, C, Fages, JG, and Lorca, X, 2017: Choco Documentation, TASC-LS2N CNRS UMR 6241, Costling S.A.S., http://www.choco-solver.org.

[11] Lesire, C and Pommereau, F, 2018: ASPiC: an acting system based on skill Petri net composition. IROS,
doi:10.1109/IROS.2018.8594328. 